\title{
Neutral beam spectroscopy for equilibrium and stability measurements for the PEGASUS toroidal experiment (abstract) ${ }^{a}$ )
}

T. Thorson, R. Fonck, and B. Lewicki

University of Wisconsin-Madison, Madison, Wisconsin 53706

(Presented on 10 June 1998)

\begin{abstract}
An optical neutral beam spectroscopy system is being designed to provide equilibrium and stability measurements for the PEGASUS toroidal experiment. Spatially localized measurements of the electron temperature and plasma density are possible by observing the intensity of the collisionally induced neutral beam fluorescence. In a helium beam, the population of the singlet levels are relatively independent of the plasma temperature compared to the triplet levels. Therefore, the ratio of intensities of a triplet transition (e.g., $3^{3} S \rightarrow 2^{3} P, \lambda=706.5 \mathrm{~nm}$ ) to a singlet transition (e.g., $3^{1} P \rightarrow 2{ }^{1} S, \lambda=501.6 \mathrm{~nm}$ ) provides a measure of the local plasma temperature for the range $10 \mathrm{eV}$ $<T_{e}<1.0 \mathrm{keV}$. The plasma density profile can be inferred from the attenuation of the beam fluorescence signal as the beam passes through the plasma. Additionally in a deuterium beam, the local plasma density can be directly inferred from the local radial gradient in the observed beam fluorescence signal. Plasma stability will be studied with localized magnetohydrodynamics measurements via beam emission spectroscopy, which otherwise is problematic for low-field spherical plasmas. (C) 1999 American Institute of Physics. [S0034-6748(99)72301-4]
\end{abstract}

a) This work was supported by U.S. Department of Energy Grant No. DE-FG02-96ER54375. 